\title{
DEMO: ourSpaces - A Provenance Enabled Virtual Research Environment
}

\author{
Peter Edwards, Chris Mellish, Edoardo Pignotti, Kapila Ponnamperuma, \\ Thomas Bouttaz, Alan Eckhardt, Kate Pangbourne, Lorna Philip, \\ and John Farrington \\ Computing Science and Geography \& Environment, University of Aberdeen, \\ Aberdeen AB24 5UA, UK \\ \{p.edwards, c.mellish, e.pignotti,k.ponnamperuma, t. bouttaz, a.eckhardt, \\ k.pangbourne, l.philip, j. farrington\} @abdn.ac.uk
}

\begin{abstract}
In this demo we present ourSpaces, a Virtual Research Environment designed to support inter-disciplinary research teams. This system has been developed to facilitate collaboration and interaction between researchers by enabling users to create, visualise and manage the provenance of research artefacts and processes.
\end{abstract}

Keywords: provenance, virtual research environment, eResearch.

\section{Introduction}

Many of the contemporary challenges facing society such as climate change require researchers from a range of disciplines to work together. Moreover, as scientific research becomes increasingly interdisciplinary in nature, the need for technologies that support collaboration and provide access to heterogeneous data and computational resources becomes ever more critical.

Some of the issues highlighted above have been explored by the PolicyGrid 1 project, a collaboration between human geographers and computer scientists as part of the UK Digital Social Research initiative. As part of this project we have developed ourSpaces2, a web-based virtual research environment which aims to provide a collaboration space for interdisciplinary academic research communities. Groups using ourSpaces work in socio-environmental and health-related domains and there are currently around 170 registered users. The system is scalable and, in principle, applicable to any research/policy domain. A screenshot of the ourSpaces web interface in presented in Figure 1.

Provenance in ourSpaces is crucial in order to support transparency and accountability of the research process by documenting the derivation history of research artefacts. The system utilizes a number of Semantic Web technologies

\footnotetext{
${ }^{1}$ This work is supported by the UK Economic \& Social Research Council (ESRC) under the Digital Social Research programme; award RES-149-25-1075.

${ }^{2}$ http://www. ourspaces.net

P. Groth and J. Frew (Eds.): IPAW 2012, LNCS 7525, pp. 198-202, 2012.

(C) Springer-Verlag Berlin Heidelberg 2012
} 
such as $\mathrm{OWI} \mathrm{L}^{3}$ and $\mathrm{RDF}_{4}^{4}$ in a user interface that shares some of the networking features of on-line social media.

\section{Provenance in ourSpaces}

At the heart of ourSpaces is a ontological framework describing different aspects of the provenance of the research process [1. An extract of this framework is illustrated in Figure 2. In order to support basic provenance we use a Web Ontology Language (OWL) representation of the Open Provenance Model [2]. This ontology defines the primary entities of OPM as well as the causal relationships that link them. OPM is a generic solution and as a result, our framework supports additional domain-specific provenance ontologies that are created by extending the concepts defined in the OPM ontology with domain-specific classes. For example, in a social simulation domain ontology one might have a Model as a type of artefact and a Simulation Experiment as a type of process. To date we have developed a number of domain-specific provenance ontologies describing aspects of Human Geography and Social Simulation. Using these ontologies it is possible, for example, to describe a physical research activity (e.g. an interview) as an opm:Process, and how such an activity causes an opm:Artifact to be generated (e.g. interview notes).

For research groups utilising ourSpaces, it is important to situate research artefacts and processes alongside people and their associated organisational structures. The current OPM specification supports limited information about a person (agent) controlling a process; there is also little regard for the wider social context. Friend-of-a-Friend 5 (FOAF) is an established RDF vocabulary for describing people and their social networks and we have opted to utilise this within our framework; a foaf:Profile is thus a subclass of the opm:Agent.

In an environment like ourSpaces, online communication is often used to comment about research artefacts or to discuss research issues. Documenting this process in the VRE it is also a crucial requirement for achieving a full and transparent provenance representation. The SIOC6 (Semantically-Interlinked Online Communities) ontology is designed to describe aspects of online communication by providing a model to express user-generated content such as posting a message in a blog or posting a comment. We have also integrated this vocabulary within our provenance framework, e.g. a sioc:post generated by a foaf : user can be associated with an opm:Artifact, opm:Process or opm: Agent.

Provenance produced in ourSpaces is stored in a repository in the form of RDF statements. Within the system we have developed a service enabling users to visualise short textual descriptions of the provenance of resources. This service translates RDF statements into English sentences, based on the approach described by Bouttaz et al. [3].

\footnotetext{
${ }^{3}$ http://www.w3.org/TR/owl-ref/

${ }^{4}$ http://www.w3.org/RDF/

${ }^{5}$ http://www . foaf-project.org/

${ }^{6}$ http://sioc-project.org/
} 


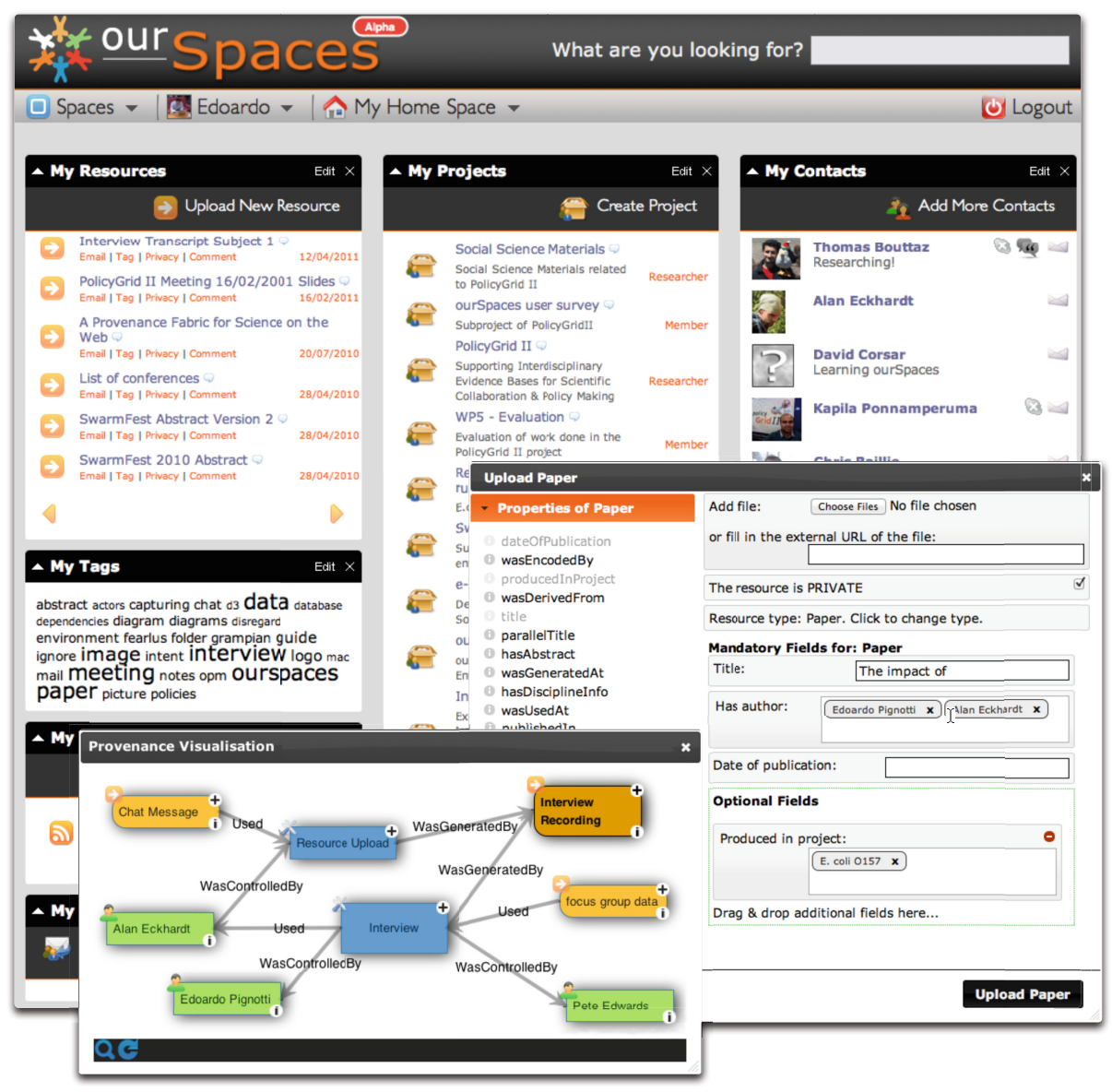

Fig. 1. A screenshot of the ourSpaces VRE showing a user's home space, an open upload form and the graphical provenance visualiser

Within the environment, there is also a need to manage users and their behaviours so that they comply with certain policies. For example, a user may impose certain access constraints on digital artefacts that he/she owns, e.g. an artefact may only be accessible to people in my social network. Provenance provides useful contextual information to trigger such policies and influence their outcome. For example, provenance would be useful in order to determine the relationship between a restricted artefact and the person requesting access to it. An artefact may only be accessible to users who are members of a particular project and who contributed towards the creation of the artefact (i.e. were named as a co-author).

We have extended our provenance framework to define such policies as a combination of conditions such as obligations, prohibitions or permissions [4]. 
We make use of the SPIN ontology [5] to support the use of the SPARQL query language to specify rules and logical constraints necessary to reason about policies. The SPIN ontology allows SPARQL queries to be represented in RDF and associated to classes in an ontology using two pre-defined description properties: spin: constraint can be used to define conditions that all members of a class must fulfil; spin:rule can be used to specify inference rules using SPARQL CONSTRUCT, DELETE and INSERT statements.

In order to support this policy framework in ourSpaces we have developed an event manager service designed to monitor events taking place in the environment, e.g. download/upload artefact, add/remove metadata, etc. When an activity is detected, the event manager initiates a policy session. The PolicyReasoner checks if any of the policies stored in the policy repository can be activated by running the SPIN reasoner against the spin:rule instances associated with the policies and stores the outcome of the activation in the policy session. In order to reason about obligation, permission or prohibition conditions we require a reasoning mechanism able to check conditions over a provenance graph. This can be seen as a semantic matchmaking problem where a functional description of a condition is matched to a subset of a provenance graph. This is done by evaluating each condition defined as a spin:rule. For an obligation, conditions have to be met; for a prohibition, the condition cannot be met; and for a permission, the condition might (or might not) be met.

Using this approach in ourSpaces we were able to implement a number of policies for use by the project teams using the system. For example, one of the policies specifies the kind of metadata required for artefacts that will be archived to the UK social science data archive - UKDA7. More specifically, a policy associated with an interview transcript requires the user to provide information about the provenance of the interview process if this is not already documented in the provenance graph. We have also implemented policies to control the re-use of resources within the VRE. In this context provenance is crucial in order to allow the creation of policies that reason about the derivation of an artefact. For example, in the map visualisation space, only publicly available data (including its sources) can be visualised as stated by the conditions of use of the OS Open Space map service 8 which is integrated into ourSpaces.

\section{Demonstration Content}

During the demonstration, we will present the ourSpaces virtual research environment. Key features of the system will be demonstrated such as creation of provenance, provenance policies, visualisation of provenance, provenance of social networks and provenance querying. A video podcast of the demo is available at: http://www.ourspaces.net/ourSpacesDemo.mov

\footnotetext{
7 http://www.data-archive.ac.uk/

8 http://openspace.ordnancesurvey.co.uk/
} 


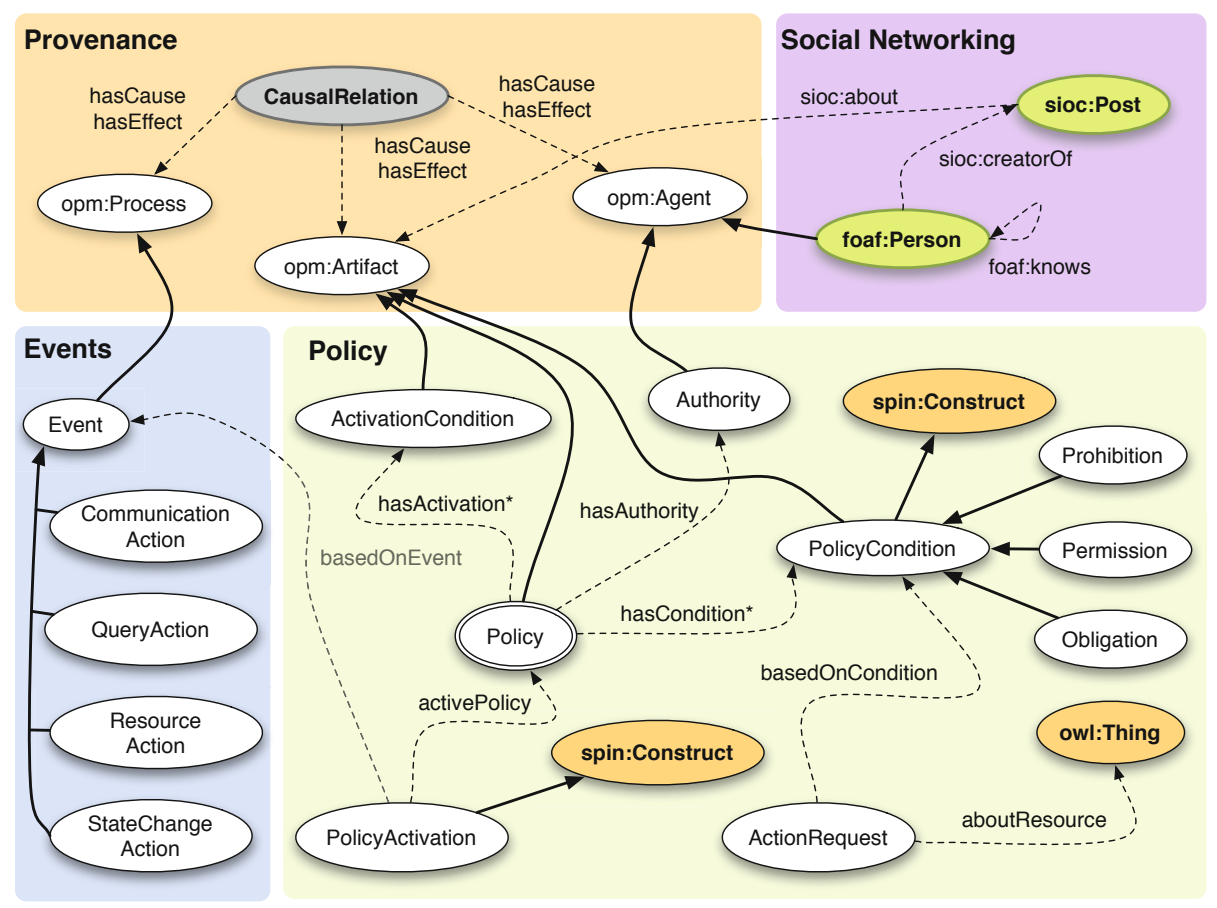

Fig. 2. An extract of the ourSpaces ontological framework

\section{References}

1. Pignotti, E., Edwards, P., Reid, R.: A Multi-faceted Provenance Solution for Science on the Web. In: McGuinness, D.L., Michaelis, J.R., Moreau, L. (eds.) IPAW 2010. LNCS, vol. 6378, pp. 295-297. Springer, Heidelberg (2010)

2. Moreau, L., Clifford, B., Freire, J., Futrelle, J., Gil, Y., Groth, P., Kwasnikowska, N., Miles, S., Missier, P., Myers, J., Plale, B., Simmhan, Y., Stephan, E., den Bussche, J.V.: The open provenance model core specification (v1.1). Future Generation Computer Systems (July 2010)

3. Bouttaz, T., Pignotti, E., Mellish, C., Edwards, P.: A policy-based approach to context dependent natural language generation. In: Proceedings of the 13th European Workshop on Natural Language Generation, Nancy, France, pp. 151-157. Association for Computational Linguistics (September 2011)

4. Pignotti, E., Edwards, P.: Using web services and policies within a social platform to support collaborative research. In: Working Notes of AAAI 2012 Stanford Spring Symposium on Intelligent Web Services Meet Social Computing (March 2012)

5. Knublauch, H., Hendler, J.A., Idehen, K.: SPIN - Overview and Motivation. Technical report, W3C Member Submission (2011) 